\title{
Los desafíos de la seguridad europea
}

\author{
Claudio Fuentes $S$.
}

Para muchos, Europa se ha convertido en una suerte de modelo de integración, por los avances que alli se han registrado. Acabada la Segunda Guerra Mundial, comenzó un proceso de acercamiento político, económico, social y de seguridad que se plasmó en el Tratado de Maastricht, en el que se planteó, entre otros, desarrollar progresivamente una política exterior y de seguridad común. En este artículo se examina la viabilidad de alcanzar una política de seguridad común dados los condicionamientos internos y externos que allí se expresan. Se analiza la institucionalidad de la seguridad europea y se especifican algunos cambios de la post-Guerra Fría. El argumento central de este trabajo es que en Europa ha sido posible avanzar en el tema de la seguridad porque existe una institucionalidad flexible que dinamiza y permite un diálogo entre actores disímiles en sus intereses y capacidades.

E1 fin de la Guerra Fría, el desaparecimiento de la Unión Soviética, la reunificación alemana y la consolidación de Estados Unidos como superpotencia militar mundial, han sido elementos determinantes para la generación de un nuevo marco de relaciones internacionales que aún no termina por definirse. A partir de 1990 desde el punto de vista institucional, Naciones Unidas se vio robustecida en su rol mediador en conflictos que han tenido consecuencias regionales (ex Yugoslavia, ex-Unión Soviética, Somalia, Ruanda) y hasta mundiales (Irak). Respecto del balance de poder, Estados Unidos se consolidó como superpotencia militar, aunque mediatizado por la solidez económica de otras regiones (Europa) o el relativo poder de determinados países (Alemania, Japón). Finalmente, el nuevo orden conllevó el resurgimiento de conflictos locales que han contribuido a generar inestabilidad en regiones específicas y que, al mismo tiempo, ponen en riesgo la efectiva aplicación de mecanismos de resolución de controversias.

En este contexto, Europa ha experimentado un cambio sustantivo en cuanto a su rol en el marco internacional. Si durante el período de Guerra 
Fría se encontraba inserta dentro del esquema de conflicto Este-Oeste, llegando incluso a delimitarse las áreas de influencia de las dos superpotencias dentro del denominado "espacio europeo", el nuevo escenario ha generado un cuestionamiento político y teórico sobre el rol que le correspondería a una Europa unida en el concierto internacional. La generación de una identidad europea respecto de otras regiones y países llevó a que en el Tratado de Maastricht se planteara como objetivo el desarrollar progresivamente una política exterior y de seguridad común.

En este trabajo se reflexiona sobre la viabilidad del objetivo europeo de contar con una política de seguridad común, dados los condicionamientos internos y externos que han venido haciéndose patentes. En la primera parte, se analiza la institucionalidad de la Seguridad en Europa. Luego se especifican algunos cambios derivados de la postGuerra Fría y, finalmente, se esbozarán algunos posibles escenarios o respuestas que surgen del proceso de integración europea, dados los condicionamientos nacionales e institucionales existentes.

\section{Institucionalidad europea.}

Existen tres instituciones de seguridad que vinculan directamente a las naciones de Europa. Es interesante observar que lo que se ha denominado la "arquitectura de seguridad europea" se compone de múltiples instancias, con distintos integrantes, funciones y características. A diferencia del proceso de integración económica, que ha tendido a la generación de organismos supranacionales para el conjunto de países integrantes de la comunidad, en el ámbito de la seguridad han subsistido en el tiempo distintas instituciones, superponiéndose muchas veces entre sí, pero que al mismo tiempo ha permitido dar al proceso una mayor flexibilidad para la resolución del conjunto de los problemas que se han enfrentado.

La Unión Europea Occidental (UEO) surgió por el Tratado de Bruselas del año 1948 -un año antes de la creación de la OTAN-y estableció los fundamentos de una organización de defensa europea incluyendo una cláusula de asistencia militar obligatoria. ${ }^{1}$ La UEO fue sobrepasada en sus funciones por la propia OTAN, minimizándose su actuación durante la Guerra Fría. Sin embargo, a mediados de los ochenta (Declaración de Roma, 1985) se activaron iniciativas en el marco de la UEO, tales como

${ }^{1}$ El Tratado de Bruselas fue firmado por Francia, Reino Unido, Bélgica, Luxemburgo y los Países Bajos. Posteriormente se incluyó Alemania, Italia, España y Portugal. 
reuniones de ministros de Relaciones Exteriores y Defensa. Posteriormente los miembros de la UEO se cuestionaron sobre el rol de Europa en el marco de la seguridad internacional, lo que se tradujo en la Plataforma de La Haya (1987) en la que se establecieron algunos criterios sobre la seguridad. Dentro de ellos, uno de los más importantes y que anticipa lo que posteriormente sería el Tratado de Maastricht, se refirió a la sentencia que una Europa integrada estaría incompleta "en tanto que su construcción no se extienda a las esferas de seguridad y de la defensa" (Montemayor, 1994).

E] Tratado del Atlántico Norte (OTAN) fue firmado en 1949 y se transformó rápidamente en una respuesta y reflejo de la bipolarización de post-guerra en torno al eje soviético y norteamericano. Básicamente la OTAN contenía el compromiso de apoyo mutuo, de defensa recíproca y de una infraestructura operacional que lógicamente pretendía responder a la amenaza militar soviética. A diferencia de otros acuerdos, la peculiaridad de este tratado es que se convirtió en una organización de carácter político y militar. La alianza Atlántica ha funcionado con un Consejo permanente y sus resoluciones han sido adoptadas por consenso de las partes. Con el paso del tiempo se consolidó como una organización con capacidad militar y de análisis, estableciendo un staff civil-militar, además de contar con una estructura militar integrada, lo que implicó contar con instalaciones de defensa comunes, ${ }^{2}$ y cuerpos militares que en tiempos de paz actúan en forma conjunta. Lo interesante de recalcar acá ha sido la capacidad de la OTAN de coordinar fuerzas armadas de los distintos países del Pacto y establecer una rutina de planificación y centralización de las decisiones en torno a los Estados Mayores Aliados (De Franchis, 1993).

Una tercera instancia de discusión de los problemas de seguridad en Europa es la Conferencia sobre Seguridad y la Cooperación en Europa (CSCE) que si bien tuvo su origen en la Guerra Fría, su propósito fue distinto a las dos instancias anteriores ya que desde el principio intentó promover el acercamiento entre las superpotencias promoviendo la cooperación y discusión de los temas de seguridad. Las negociaciones para la creación de este foro se iniciaron en los años sesenta y se consolidaron a partir de 1973 en una conferencia que dio por resultado el Acta de Helsinki de agosto de 1975 (Ghebali, 1989). Desde aquella fecha la CSCE ha sido el único foro que reunió a países europeos

\footnotetext{
${ }^{2}$ En el caso de la estructura militar participan todos los miembros de la OTAN (16 en total) con la excepción de Francia, España e Islandia.
} 
occidentales, orientales, neutrales y no alineados llegando a contar a la fecha con 52 miembros.

Del sumario análisis de esta institucionalidad pueden observarse la superposición de organismos y funciones. Esto encuentra su origen en que cada uno de ellos ha surgido en coyunturas específicas de la Europa comunitaria. Aunque aparentemente este hecho pudo ser factor de entrabamiento de ciertos procesos, la dinámica propia de la Guerra Fría requería de organizaciones que fuesen flexibles a las particulares circunstancias de tensión Este-Oeste, que admitieran amplios grados de consenso y que no dejaran cerradas las puertas a la cooperación interbloques (CSCE). De este modo, logró equilibrarse la participación de visiones distintas respecto de la seguridad (Francia-Estados Unidos por ejemplo), y en un marco de cooperación que crecientemente se tornó más eficiente (recuérdense las negociaciones OTAN-Pacto de Varsovia y los acuerdos de reducción de armas Estados Unidos-Unión Soviética en la década de los ochenta).

\section{La post Guerra Fría y la nueva institucionalidad europea.}

El colapso de la Unión Soviética y los cambios que se experimentaron en la región rápidamente provocaron una redefinición de los parámetros de la seguridad europea. En la Carta de París para la nueva Europa (21 de noviembre, 1990) se institucionalizó la CSCE pero se advirtió que dicho organismo no podía ser el instrumento para controlar los cambios, y ello debido a dos razones fundamentales: primero, porque la CSCE tenía facultades de carácter más declaratorio que operacional; y, segundo, que este organismo tenía que competir con otras instituciones internacionales como OTAN, Comunidad Europea, UEO y el Consejo de Europa. Sin embargo, la discusión de la redefinición del ámbito de la seguridad estaba presente $y$, posteriormente, se desarrollaría en el Tratado de Maastricht de febrero de 1992 y en el documento final de Helsinky de mediados de ese mismo año.

Sin duda el Tratado de Maastricht constituyó un salto cualitativo por cuanto la Unión Europea alude explícitamente a la creación de una Política Exterior y Seguridad Común (PESC). Cabe recordar que en 1969 los Estados miembros de la comunidad habían incluido una dimensión política a la cooperación a través de la Cooperación Política Europea 
(CPE) que se tradujo en reuniones periódicas de los ministros de Asuntos Exteriores, culminando en el Acta Unica Europea de 1987 (AUE).

Dada la cohesión alcanzada entre los Estados miembros de la actual Unión, en Maastricht se consolida la noción de contar con una política exterior y de seguridad común, aunque se especifica que sería una actividad intergubernamental tal como fue la CPE. Las decisiones tendrían que ser adoptadas por unanimidad, aunque algunas decisiones específicas podrían adoptarse por mayoría. Correspondería a la Comisión participar de los trabajos de política exterior y de seguridad común, disponiendo de un derecho de iniciativa que es novedoso en la Unión. Además, se acordó el estructurar o elaborar una política de defensa común e integrar más armónicamente la UEO al proceso de toma de decisiones de la comunidad.

Claramente una de las dificultades de la adopción de decisiones en materia de seguridad y política exterior se refiere a la falta de consenso entre los Estados miembros. El Tratado estipula que las líneas fundamentales de estas políticas correspondería definirlas al Consejo Europeo de Jefes de Estado o Gobierno por unanimidad, dando la facultad al Consejo de decidir algunas materias por mayoría cualificada. En este mismo sentido y para facilitar las decisiones, los Estados en la medida de lo posible renunciarían al veto.

Dentro de los temas definidos por el Tratado de carácter común a sus miembros, figuran: la no proliferación de armamentos nucleares; el control de la transferencia de armamentos y de tecnología a terceros países, el control de las exportaciones de armas; el desarme y el control de armamento en Europa; y, el proceso de la CSCE.

Profundizando los elementos presentes en Maastricht, en la Cumbre de Helsinky de 1992, se especifican algunos de los temas ya señalados, a través del Documento Final titulado "El desafío del cambio" (10 de julio 1992). ${ }^{3}$ En este se introducen al menos tres nuevos elementos: una definición del concepto de seguridad global; la atribución a la CSCE de medios operacionales para la prevención y solución de conflictos; y, el establecimiento de un Foro de Cooperación en Materias de Seguridad.

El primer hecho interesante es que en Helsinky los Estados acuerdan una nueva conceptualización de seguridad, entendiéndola como de carácter global, cooperativa, múltiple, indivisible y con vinculaciones mundiales, regionales y locales. Los Estados al optar por la premisa de

${ }^{3}$ Una versión del Documento en: Desarme, N4, Revista de Naciones Unidas, 1992, pp.195 y ss. 
igualdad de derechos, no pueden reforzar su seguridad en detrimento de otros Estados por lo que se asienta el carácter cooperativo, creándose el Foro de Cooperación en Materia de Seguridad. Por otra parte, la seguridad se entiende en su globalidad e indivisibilidad vinculando directamente aspectos relacionados con el mantenimiento de la paz internacional, el respeto de los derechos humanos, la democracia, el desarrollo económico, la justicia social, la protección del medio ambiente y todos los aspectos que afecten de algún modo la estabilidad de las sociedad democráticas. Finalmente, al considerar las vinculaciones mundiales, regionales y transfronterizas de la seguridad, se entendía el fortalecimiento de la cooperación con Naciones Unidas en lo concerniente a la prevención, gestión y solución de conflictos (Ghebali, 1992).

El segundo elemento presente en Helsinky se refirió a los medios operacionales, lo que de hecho implicó un salto cualitativo en relación a su anterior configuración. Aunque con una gran dosis de realismo, se consideró que la CSCE debía transformarse en un instrumento para controlar los efectos positivos y negativos de los cambios que se sucedían en la región, y de ahí la necesidad de generar instancias que efectivamente permitiesen prevenir y gestionar la crisis y resolver pacíficamente las controversias. De esta forma, se propuso la consolidación de un Comité de Altos Funcionarios (CAF), la creación de un Alto Comisionado para las Minorías Nacionales, y la autorización a la CSCE a participar en actividades de mantenimiento de la paz. Este último aspecto tiene importancia pues la transforma en una institución auténticamente operacional.

El tercer elemento del documento se refirió al establecimiento de un Foro de Cooperación en Materia de Seguridad abierto a los miembros de la CSCE encargado de tres funciones inmediatas: negociación, diálogo constante y reflexión sobre el tema de la prevención de los conflictos. En términos prácticos a través del Foro se permitiría la negociación de medidas concretas relativas al desarme; proporcionar un marco para consultas, cooperación y diálogo; y, analizar de manera pormenorizada el tema de la prevención de conflictos, aunque no avanza mucho en la discusión sobre la creación de un Centro para la Prevención de Conflictos establecido en la CSCE en 1991.

La crisis en la ex-Yugoslavia demostró nútidamente las dificultades de los aparatos institucionales para generar políticas consensuales en materia de seguridad. De hecho, una de las principales críticas a la CSCE 
se refiere precisamente a su sistema de toma de decisiones ya que al ser de carácter consensual (unanimidad de sus miembros) y contar con 52 miembros, muchas veces la Conferencia entorpece y hasta provoca una paralización de las discusiones y decisiones que paradójicamente, son las que más rapidez requieren. Una segunda crítica se refiere al mal equipamiento de la CSCE para la solución pacífica de controversias. Algunos países como Francia han propuesto la creación de un tribunal de conciliación y arbitraje. Por su parte, Estados Unidos ha propuesto la generación de formas de conciliación entre las partes en conflicto a través de la CSCE (Ghebali, 1992; Torstila, 1992).

Helsinki 92 no puede entenderse como un intento de conformar una alianza militar, sino que está orientado hacia lo que se ha denominado la creación de un "sistema de seguridad cooperativa" en la que, sin romper con los dispositivos nacionales de defensa, se tenga por objetivo garantizar la seguridad en conjunto, cooperativamente y respaldándose mutuamente (Torstila, 1992).

\section{Los desafios de la seguridad común (¿es posible?).}

El cambio de escenario en Europa constituye un elemento de incertidumbre para responder adecuadamente a la pregunta de si es posible la estructuración de una seguridad común en Europa. Durante la Guerra Fría existía claridad respecto a los objetivos de las instituciones que ya hemos analizado: defensa de intereses regionales (UEO), defensa de intereses occidentales (OTAN), o alternativa de diálogo interbloques (CSCE). El nuevo escenario internacional no presenta las mismas condiciones por lo que la institucionalidad existente ha comenzado ha modificar sus tradicionales visiones y acciones.

Las principales dificultades que se observan para lograr el objetivo de seguridad común se refieren a las diferentes visiones entre los Estados miembros de la Unión; la inestabilidad de Europa del Este y la redefinición de un nuevo espacio europeo; y, el rol de Estados Unidos en Europa.

La crisis yugoslava fue una lección importante para Europa pues demostró que ante una situación cercana, conflictiva y de grandes costos para la estabilidad regional, no existían intereses comunes entre los miembros de la Unión. El establecimiento de una política exterior y de seguridad común necesariamente requiere de la existencia de una comunalidad de intereses en relación al sistema internacional. Al no existir 
esa condición básica, se dificulta el concordar políticas comunes al conjunto de Estados miembros y, al mismo tiempo, se evidencia la necesidad de contar con instrumentos para la prevención de conflictos que dejan poco espacio para negociaciones prolongadas por la rapidez de su desarrollo y que demandan altos grados de coherencia e eficacia intergubernamental (Jopp, 1994).

La misma dificultad en la ratificación del Tratado de Maastricht demostró que la Unión Europea no es una entidad homogénea ya que subsisten distintas visiones sobre el objetivo final de dicha Unión. Al intensificarse el proceso se evidenció con mayor notoriedad la tensión entre Estado-nación e integración transnacional. Particular importancia adquiere la discusión sobre la soberanía nacional y comunitaria más cuando se trata de asuntos que afectan el concepto de "identidad nacional", de los países miembros.

Una segunda dificultad se refiere a la integración de los países del Este al esquema de seguridad occidental. La discusión de ese tema se ha centrado en la posible ampliación de la OTAN hacia los países del Este, cuestión que aparece difícil en el corto plazo. No obstante, como una forma de ampliar la participación a los nacientes Estados del Este, en 1991 se creó el Consejo de Cooperación del Atlántico Norte (CCAN) que pretendía ser un nuevo foro para la cooperación y la consolidación de las democracias del Este. Para Europa occidental existen dos alternativas en relación con los vecinos del Este: incorporarlos a los esquemas de seguridad occidental o bien, integrar a los Estados paso a paso, de acuerdo a su incorporación a la Unión Europea. El desafío es generar condiciones de gobernabilidad y estabilidad en las naciones del Este y para ello el camino elegido hasta el momento ha sido la ampliación de los foros occidentales (CCAN, CSCE) para generar oportunidades de diálogo internacional.

Un caso que ilustra la alta sensibilidad de la zona europea del Este se refirió a las informaciones provenientes de Rusia en las que se señalaba el traspaso de tecnología nuclear a Irán. ${ }^{4}$ Esto último, unido con la actuación rusa en Chechenia, nos indican la importancia que para Europa tiene consolidar un entorno relativamente estable.

La tercera dificultad de la seguridad común europea es el rol que juega Estados Unidos en el concierto internacional. En este sentido, la OTAN contribuye a consolidar la presencia de Estados Unidos en la región. Por

\footnotetext{
${ }^{4}$ El Financiero, México, 17 de marzo de 1994.
} 
su parte, la UEO en la medida en que ha consolidado una presencia propiamente europea más que "occidental", tiende a convertirse en lo que se ha llamado el "pilar europeo" dentro de la OTAN (Montemayor, 1994). Más que apuntar a la generación de entes competitivos, lo que se ha demostrado en este tiempo de transición ha sido la necesaria coexistencia de múltiples instancias en donde se han buscado consensos y se ha reflexionado sobre las necesarias adecuaciones institucionales a la nueva realidad internacional (Gebhard, 1994). La discusión en la OTAN se ha orientado al debate sobre una nueva estrategia y estructura de fuerzas adecuada para el manejo de crisis y mantenimiento de la paz, la preocupación en los problemas de Europa del Este y su evolución, y la cooperación de la OTAN con instituciones como Naciones Unidas, UEO y CSCE. En tanto, en Europa propiamente tal, la discusión se ha centrado en el fortalecimiento del la PESC del Tratado de Maastricht y en la vinculación europea a instancias tales como la CSCE y la OTAN. Lo importante de subrayar es que la OTAN es la única organización que cuenta con un poderío militar eficiente; la UEO es la única organización que representa al núcleo de identidad europea en materia de seguridad; $y$, finalmente, la CSCE es el único foro abierto a la totalidad de los países europeos.

La discusión europea sobre seguridad, aunque con mayor cautela que en otros ámbitos, reproduce la divergencia entre quienes defienden la búsqueda de una mayor identidad Europea, y aquéllos que prefieren una vinculación sólo intergubernamental, sin repercusiones supranacionales.

Las consideraciones anteriores permiten aventurar la mantención de un relativo statu quo institucional en materia de seguridad, con una mayor interconexión entre las instancias internacionales de seguridad ya mencionadas. La teoría de los círculos concéntricos (UEO 9 miembros, OTAN 16 miembros, CSCE 52 miembros) parece ser una respuesta flexible y que permite una interconexión entre países que si bien tienen el interés común de preservar la paz, no necesariamente están de acuerdo en la forma de concretarlo (Pinheiro, 1991). En el ámbito de la seguridad se confirma lo que algunos estudiosos han denominado la "geometría variable" de la Unión ya que subsisten distintos grados de acercamiento y se proyecta una diversidad de objetivos entre los Estados miembros. Lo interesante de destacar en este análisis es que en la medida en que surgen escenarios de riesgo extra Unión Europea, se dinamizan simultáneamente los espacios de diálogo político en las tres instancias analizadas. 
Pero además, la teoría de los círculos concéntricos admite una distinción entre los países miembros de la Unión Europea que favorezcan una rápida integración de la PESC y aquellos que se muestren más reticentes a ello. La conformación de un cuerpo militar conjunto franco-alemán fue un síntoma de la diversidad de opiniones dentro de la Unión. De este modo, la tendencia es hacia la profundización de las vinculaciones entre algunos países en materias de seguridad; la ampliación a nuevos miembros a través de instancias de diálogo y cooperación intergubernamental y el mantenimiento de equilibrios extraregionales a través acuerdos ya consolidados en el tiempo (OTAN).

En el caso específico de la Unión Europea se presenta una dificultad entre quienes forman parte de la UEO, aquellos que adhirieron al Tratado de Maastricht y los que forman parte de la OTAN. En la medida en que se consolide la PESC del Tratado de Maastricht tenderá a diluirse aquella contradicción, y por lo mismo, tenderá a fortalecerse la noción de un "brazo político" de la Unión Europea en el marco de la OTAN. Sin embargo, y allí está la esencia del actual debate sobre seguridad, el Tratado de Bruselas de 1948 que creó la UEO expira en 1998 a menos que los Estados miembros renueven su vigencia. Las alternativas son institucionalizar la PESC por acuerdo de los 15 miembros de la Unión, o bien, renovar la vigencia de la UEO por acuerdo de algunos miembros de la Unión, manteniendo una estructura similar a la actual.

El desarrollo y resolución de este debate entregará muchos elementos sobre la profundidad de intenciones de los Estados partícipes de la Unión Europea, en consolidar verdaderamente una noción de seguridad común. Aceptando la diversidad de criterios existente, no resultaría ilógico predecir que se buscaran fórmulas consensuales que incorporarán, en distintos niveles, la participación de diversos actores de la región.

Quizás_el_principaléxito_de la_Europa comunitaria en el tema_de la seguridad haya sido el no cerrarse a estructuras inflexibles, sino que por el contrario, siempre ha buscado -bajo distintas fórmulas institucionales- alcanzar el objetivo de la paz. Es en aquella flexibilidad donde encontramos su mayor virtud y por ello no parecería extraño que se verificaran en el corto y mediano plazo avances graduales, pero muy significativos, en materia de seguridad, todos con el objetivo común de brindar seguridad a una Europa interdependiente. 


\section{Bibliografía}

Buzan, Barry, et al., The European Security Order Recast. Scenarios for the Post Cold War Era. Copenhagen: Centre for Peace and Conflict Research, 1990.

De Franchis, Amadeo, "La Seguridad Global en el Escenario de Post-Guerra Fría", en: Argentina-Otan. Perspectivas sobre la Seguridad Global. Argentina: CARI, GEL, 1993.

Duret, José Manuel, Estatuto de fuerzas armadas OTAN y España. España: Biblioteca TECNOS, 1982.

Gebhard, Paul, "The United States and European Security", Adelphi Paper, N2286, February, 1994.

Ghebali, Victor-Yves, "Hacia una institución operacional encargada de la Seguridad Global", Desarme, N4, Revista de Naciones Unidas, 1992.

Gomariz, Enrique, "Las medidas de confianza mutua en la Europa de fin de siglo", en: Augusto Varas e Isaac Caro (eds.), Medidas de Confianza Mutua en América Latina. Santiago de Chile: FLACSO, Stimson Center, SER, 1994.

Jopp, Mathias, "The Strategic Implications of European Integration", Adelphi Paper, N290, July, 1994.

Lutz, Dieter, "Hacia un sistema de seguridad colectiva Europea", Desarme, N ${ }^{\circ} 4$, Revista de Naciones Unidas, 1992.

Mirello, Nelson, Sistemas Militares Internacionales: LA OTAN y el Pacto de Varsovia. Grandes Tendencias Contemporáneas. México: UNAM, 1986.

Montemayor, Vicente, "La nueva arquitectura de seguridad colectiva europea", Relaciones Internacionales, N61, Revista Facultad de Ciencias Políticas, UNAM, enero-marzo 1994, pp.37 y ss.

Pinheiro, Joao de Deus, "La arquitectura europea de Seguridad", Revista de la OTAN, $\mathrm{N}^{\circ} 1$, febrero 1991.

Salvadori, Massimo, OTAN, La comunidad del Atlántico Norte. Argentina: Colección Hombres y Problemas, 1958.

Torstila, Pertti, "El éxito del proceso de Helsinki y los nuevos retos", Desarme, $\mathrm{N}^{\circ} 4$, Revista de Naciones Unidas, 1992. 
Tratados y Documentos revisados:

- Tratado de Maastricht, 1992.

- Documento Final Cumbre de Helsinky, 1992.

- Carta de París para la Nueva Europa, 1990.

- Tratado de Bruselas, 1948. 